\title{
Neuro-oncology family caregivers' view on keeping track of care issues using eHealth systems: it's a question of time
}

\author{
Florien W. Boele ${ }^{1,2,3}$ (D) Cornelia F. van Uden-Kraan ${ }^{4} \cdot$ Karen Hilverda $^{5}$. \\ Jason Weimer ${ }^{1} \cdot$ Heidi S. Donovan ${ }^{1} \cdot$ Jan Drappatz $^{6} \cdot$ Frank S. Lieberman $^{6}$. \\ Irma Verdonck-de Leeuw ${ }^{4}$ Paula R. Sherwood ${ }^{1}$
}

Received: 4 January 2017 / Accepted: 20 May 2017 / Published online: 26 May 2017

(c) The Author(s) 2017. This article is an open access publication

\begin{abstract}
Primary brain tumors (PBTs) are rare but have a great impact on both patient and family caregiver wellbeing. Supporting caregivers can help them to continue their caregiving activities to maintain the patients' best possible level of quality of life. Efforts to improve PBT caregiver wellbeing should take into account country- or culture-specific differences in care issues and supportive care needs to serve larger caregiver groups. We aimed to explore PBT caregivers' satisfaction with the current supportive care provision, as well as their thoughts on monitoring their care issues with both paper-based and digital instruments. Twelve PBT caregivers were interviewed in the United States. The semi-structured interviews were transcribed verbatim and analyzed by two coders independently. Data were combined with those collected in the Netherlands, following similar methodology $(N=15)$. We found that PBT caregivers utilize both formal and informal
\end{abstract}

Florien W. Boele

F.Boele@leeds.ac.uk

1 School of Nursing, University of Pittsburgh, 336 Victoria Bldg, Pittsburgh, PA 15261, USA

2 Leeds Institute for Cancer and Pathology, St James's Hospital, University of Leeds, Beckett Street, Leeds LS9 7TF, UK

3 Leeds Institute of Health Sciences, University of Leeds, Worsley Building, Clarendon Way, Leeds LS2 9NL, UK

4 Department of Clinical, Neuro and Developmental Psychology, VU University, Van der Boechorststraat 1-3, 1081BT Amsterdam, The Netherlands

5 Department of Medical Psychology, VU University Medical Center, PO Box 7057, 1007MB Amsterdam, The Netherlands

6 Division of Neuro-Oncology, Hillman Cancer Center, University of Pittsburgh Medical Center, 5115 Centre Avenue, Pittsburgh, PA 15232, USA support services, but that those who experience more care issues would prefer more support, particularly in the early disease phase. Keeping track of care issues was thought to provide more insight into unmet needs and help them find professional help, but it requires investment of time and takes discipline. Caregivers preferred a brief and easy-touse 'blended care' instrument that combines digital monitoring with personal feedback. The present study shows that the preferences of family caregivers in neuro-oncology toward keeping track of care issues are likely not heavily influenced by country- or culture-specific differences. The development of any instrument thus has the potential to benefit a large group of family caregivers.

Keywords Family caregiver · Brain tumor · Neurooncology $\cdot$ Supportive care $\cdot$ eHealth

\section{Introduction}

The diagnosis and treatment of a primary brain tumor (PBT) can have a devastating effect on the patient and their family. With the prognosis and treatment options being highly dependent on the tumor type and grade, and many tumors eventually recurring or progressing [1], dealing with uncertainty of the future is a major issue for most patients [2]. Moreover, disease-specific symptom burden can include debilitating physical and neurological sequelae such as paresis, sensory loss, visual-perceptual deficits, cognitive deficits, and seizures $[3,4]$. Changes in personality and behavior, fatigue and mood issues also occur frequently $[5,6]$. Together, these symptoms can hinder patients' ability to function independently and influence their quality of life (QOL) as well as the QOL of their family members. 
Family members and close friends commonly provide patients with requisite physical and emotional support, and are therefore called 'family caregivers'. This can be a rewarding task, but many family caregivers also report considerable burden and distress as a result of providing care [7]. Not only may the psychological and behavioral responses to caregiving influence caregivers' emotional and physical health [8], it may also influence the quality of care delivered to the patient at home. Professional support in meeting the needs of family caregivers to decrease their distress, is imperative to enable them to continue their caregiving activities and maintain the best possible level of patients' wellbeing.

Frequent monitoring with patient reported outcomes (PROs) to track needs over time, paired with feedback and followed up with supportive care as needed has been suggested as a possible way to meet patients' needs [9]. The same principle may be applied to caregivers in meeting their own needs. This monitoring may be introduced into clinical practice through administering paper questionnaires, or by means of computerized or web-based (eHealth) applications. With the growing popularity of eHealth and ongoing advances in technology, applications are sometimes developed before consulting end users' opinions on their preferences [10]. This can harm the effectiveness and uptake of eHealth solutions and indeed, it has been suggested that engaging the family caregivers as end users in the development of any pathway to resolve supportive care needs is vital to improve its usefulness in practice $[10,11]$.

In our previous report on the attitudes and preferences of Dutch glioma patients and their family caregivers regarding monitoring symptoms, distress and QOL issues, we found that although the preferred method for monitoring issues is highly personal, a combination of online and personal care ('blended care') was advised [12]. Importantly, we found that family caregivers viewed monitoring symptoms, distress and QOL (collectively called 'care issues' hereafter) more favorably than patients did. Details on patients' and caregivers' perceived (dis)advantages for keeping track of symptoms, distress and QOL issues were provided and pros and cons for both paper-based and computer-based methods were discussed. However, our previous report lacks details needed to develop or adjust monitoring systems to best meet PBT caregivers' preferences and needs. Moreover, there may be country or culture specific differences in the expression of distress, QOL, or supportive care needs [13], as well as differences in the organization of health care and accessibility of supportive care between different countries. As PBTs are rare and the needs of the family caregivers do not always overlap with those of other oncological caregiving populations, it is vital that any instrument developed has the potential to benefit as many PBT caregivers as possible.

Therefore, we now add data from a sample of neurooncology caregivers from the United States and reexamine the transcripts from the Dutch caregiver interviews. Specifically, the purpose of this study is to investigate in detail how to adjust monitoring care issues to the preferences of family caregivers in neuro-oncology in general by focusing on: (1) experiences with current supportive care provision; (2) caregivers' ideas on how to make monitoring care issues more acceptable; (3) and their preferences, as well as the perceived (dis)advantages of paper-based and digital monitoring instruments. Recommendations will be made which can contribute to the future development of instruments to monitor PBT caregiver issues.

\section{Methods}

\section{Sample and procedure}

Caregivers were recruited at the outpatient department of a NCI-designated Cancer Center between August and November 2015. Caregivers were eligible if they (1) were $>18$ years old; (2) were the primary family caregiver of a patient diagnosed with a PBT (any type). Caregivers could not participate if they did not provide informed consent or if they had insufficient understanding of the English language to provide consent and/or complete the interview. Additionally, caregivers could not participate if the patient did not provide informed consent for the caregiver to be interviewed, or if they lived further than a $3 \mathrm{~h}$ drive from the Cancer Center and were unable to be interviewed at the hospital. The treating physician, physician's assistant, or nurse practitioner introduced potential participants to the study and obtained permission to be approached by a member of the research team, who then contacted them regarding their willingness to participate. Purposive sampling was done to ensure participants represented the general caregiving population.

Interviews were semi-structured and lasted for about 60 min, taking place either in the hospital or at a location of the participant's preference. Interviews were performed until data saturation occurred, meaning that no new information was generated by the last interview(s) [14]. All interviews were recorded. Data were combined with those collected during a previous study performed in the Netherlands, following similar methodology [12]. All participants signed written, informed consent forms and the study protocols were approved by the University of Pittsburgh (PRO15040347) and the Institutional Review Board of the VU University Medical Center (protocol number 13/309). 


\section{Interviews}

Interviews were introduced to participants by explaining that we were interested in their care issues, their experiences with supportive care, and their thoughts and ideas regarding different methods to keep track of their care issues over time, so that issues may be recognized and information and support may be accessed in a timely manner. The same semi-structured interview schedule from our previous publication was used [12]. To summarize, the main topics were (1) experiences with supportive care provision and unmet supportive care needs; (2) thoughts and suggestions regarding keeping track of care issues, including frequency, timing and format; (3) attitudes toward and conditions for use of different paper-based (the Distress Thermometer [15]) and eHealth instruments (Oncoquest [16] and Oncokompas [17]) to track care issues over time. Although these instruments are all patient-oriented, we felt these were suitable examples to show caregivers because they rely on technology in various degrees: from no technology being used with the distress thermometer (DT), to Oncoquest $(\mathrm{OQ})$ which is part computer-based and part comprised of personal contact, to Oncokompas (OK) which is completely computer-based. Also, the degree to which health care professionals would be involved varies: the DT and OQ instruments would ideally be fed back by a nurse, who would then initiate referral if needed, whereas OK is a self-management tool. Participants were shown printed pictures and screen shots accompanied by English translations where appropriate, and detailed verbal explanation of the instruments was provided. Prompts were offered if open-ended questions elicited little response. Interviews were performed by a research psychologist (FWB)—or, in one case, by a $\mathrm{PhD}$-student with experience in interviewing for qualitative analysis and supervised by FWB (NSK; acknowledgments).

\section{Data analysis and reporting}

The interviews were transcribed verbatim. Thematic data analysis [18, 19], similar to the methods used in our previous publication [12], was conducted by two coders (PRS and FWB). The coders read the transcripts several times to familiarize themselves with the content, and highlighted sections of the transcripts that were related to the research objectives and independently selected and coded these into key issues and themes. The coders met frequently to discuss their findings, refine the key issues and themes, and resolve possible differences until consensus was reached. If needed, a third researcher (CFU) was available to guide this process. Finally, one coder (FWB) examined all transcripts again, including the transcripts from the Dutch interviews, to ensure that the analytical process was robust. Where applicable differences between the US and Dutch caregiver samples are highlighted in the Results. The consolidated criteria for reporting qualitative research (COREQ) [20] were used in this report. All quotes provided were anonymized.

\section{Results}

\section{Participants}

Twenty-three caregivers were invited, of whom 12 agreed to participate. Reasons for non-participation were: not interested $(N=5)$; too busy $(N=2)$; unable to reach by phone to schedule interview $(N=2)$; declined home visit and interview could not be scheduled in the hospital due to time constraints $(N=1)$. Data saturation was reached with the 12th participant. The sample is representative of caregivers of patients with a wide range of neuro-oncological diagnoses across different disease stages; see Table 1 for characteristics of caregivers from the present sample and the previously collected Dutch sample.

\section{Experiences with supportive care for caregivers}

The support family caregivers reported to have received or have been offered can be categorized into:

(1) Formal support from health care and religious services; (2) informal support from friends, family and community members; and (3) support from work, see Table 2. Formal support services included support from specialist nurses either through the neuro-oncology clinic, psychiatry or primary care physician's office; social work or psychology services; support groups for caregivers; practical support such as meals on wheels; and medication (e.g. antidepressants, sleep medication). US caregivers specifically frequently mention religious services such as pastoral counseling, and only Dutch caregivers report having received legal advice, help with completing tax forms in light of their special circumstances and transportation services to and from the hospital.

Examples of informal support provided include social support such as being there in the hospital, supporting each other through tough times; practical support such as people coming over to bring dinner, deliver groceries, or helping out with other chores; and financial support such as helping to pay for medical bills or housekeeping. Particularly practical and financial support were mentioned more often by US caregivers, e.g.:

'They took up a collection through a variety of different things. They had a housekeeper come in and clean 
Table 1 Demographic characteristics of participants
US sample $(N=12)$

Dutch sample $(N=15)$

\begin{tabular}{|c|c|c|}
\hline \multicolumn{3}{|l|}{ Age } \\
\hline $30-40$ & $2(16.7 \%)$ & $1(6.7 \%)$ \\
\hline $40-50$ & $2(16.7 \%)$ & $3(20 \%)$ \\
\hline $50-60$ & $3(25 \%)$ & $9(60 \%)$ \\
\hline $60-70$ & $4(33.3 \%)$ & $1(6.7 \%)$ \\
\hline $70-80$ & $1(8.3 \%)$ & $1(6.7 \%)$ \\
\hline \multicolumn{3}{|l|}{ Sex } \\
\hline Male & $6(50 \%)$ & $2(13.3 \%)$ \\
\hline Female & $6(50 \%)$ & $13(86.7 \%)$ \\
\hline \multicolumn{3}{|l|}{ Relationship to patient } \\
\hline Spouse & $10(83.3 \%)$ & $15(100 \%)$ \\
\hline Parent & $1(8.3 \%)$ & $0(0 \%)$ \\
\hline Cousin & $1(8.3 \%)$ & $0(0 \%)$ \\
\hline \multicolumn{3}{|l|}{ Diagnosis of patient } \\
\hline Oligodendroglioma grade II & $2(16.7 \%)$ & $3(20 \%)$ \\
\hline Astrocytoma grade II & $1(8.3 \%)$ & $1(6.7 \%)$ \\
\hline Oligodendroglioma grade III & $0(0 \%)$ & $3(20 \%)$ \\
\hline Astrocytoma grade III & $1(8.3 \%)$ & $2(13.3 \%)$ \\
\hline Glioblastoma grade IV & $6(50 \%)$ & $7(46.7 \%)$ \\
\hline Medulloblastoma & $1(8.3 \%)$ & $0(0 \%)$ \\
\hline Meningioma & $1(8.3 \%)$ & $0(0 \%)$ \\
\hline \multicolumn{3}{|l|}{ Time since diagnosis } \\
\hline$<1$ year & $1(8.3 \%)$ & $5(33.3 \%)$ \\
\hline $1-2$ years & $5(41.7 \%)$ & $1(6.7 \%)$ \\
\hline $2-3$ years & $2(16.7 \%)$ & $3(20 \%)$ \\
\hline $3-4$ years & $1(8.3 \%)$ & $0(0 \%)$ \\
\hline $4-6$ years & $0(0 \%)$ & $1(6.7 \%)$ \\
\hline$>6$ years & $3(25 \%)$ & $5(33.3 \%)$ \\
\hline \multicolumn{3}{|l|}{ Disease status } \\
\hline Stable disease & $2(16.7 \%)$ & $10(66.6 \%)$ \\
\hline Disease progression (suspected) & $4(33.3 \%)$ & $2(13.3 \%)$ \\
\hline Under treatment & $6(50 \%)$ & $3(20 \%)$ \\
\hline
\end{tabular}

our house for 6 months. For 6 months! They paid for it. [...] It was tremendous.'

Husband of GBM patient who is currently under treatment

Of note, two caregivers expressed that too much informal involvement can be a burden as well, as continually having to explain to family and friends that the patient is not well despite their healthy appearance and the lack of time to be together as a couple can be difficult. Under support from work, many caregivers reported their employers had been very understanding and allowed them to cut back on hours and/or increase hours as needed, and some mention having been given the option to work from home.

In a few cases, questionnaires were used in the clinic to screen for needs. However, none reported to have received any feedback and if needs were identified, there was no further follow-up or referral to professional services. When asked if they felt the supportive care offered was sufficient, caregivers with few care issues were generally satisfied while others indicated they would have liked more information on how to deal with the patient's symptoms and prognosis, as well as more practical, financial and social support especially early in the disease trajectory.

'Oh I think we need something! We're not professionals, we don't know what we're doing. [...] Are we making it better, are we making it worse? We don't know, we've not been in this situation before.'

Cousin of meningioma patient with suspected disease progression

Common barriers to supportive care services were: support was only offered if actively requested; support was not offered when caregivers and patients appeared to 
Table 2 Caregivers' experiences with supportive care

\begin{tabular}{|c|c|c|c|}
\hline Topics & Key issues & Themes & Participant groups \\
\hline \multirow[t]{12}{*}{ Formal support } & \multirow[t]{6}{*}{ Emotional support } & Nurse(-specialist) & Both groups \\
\hline & & Psychologist & Both groups \\
\hline & & Social worker & Both groups \\
\hline & & Peer support group & Both groups \\
\hline & & Medication (e.g., antidepressants, sleeping pills) & Both groups \\
\hline & & Religious services & US group \\
\hline & \multirow[t]{5}{*}{ Practical support } & Nurse(-specialist) & Both groups \\
\hline & & Meals on wheels & US group \\
\hline & & Transportation service to/from the hospital & Dutch group \\
\hline & & Legal advice, e.g., preparing a will & Dutch group \\
\hline & & Help with completing tax forms & Dutch group \\
\hline & Needs screening in clinic & Questionnaires in clinic without feedback or referral & Both groups \\
\hline \multirow[t]{15}{*}{ Informal support } & \multirow[t]{6}{*}{ Social support } & Family and friends being there in the hospital & Both groups \\
\hline & & Supporting each other through tough times & Both groups \\
\hline & & Family and friends watching the patient when the caregiver's away & US group \\
\hline & & Family or friends setting up Facebook account to provide support & Both groups \\
\hline & & Family member coming back to live at home and provide support & US group \\
\hline & & Community members praying for patient and caregiver & US group \\
\hline & \multirow[t]{6}{*}{ Practical support } & Making dinner, bringing food over to the house & US group \\
\hline & & Getting groceries & US group \\
\hline & & Helping with household chores & US group \\
\hline & & Helping the patient get ready for the day & US group \\
\hline & & Providing a wake-up call service & US group \\
\hline & & Friend with medical background would answer questions & US group \\
\hline & \multirow[t]{3}{*}{ Financial support } & Taking up a collection to support a housekeeper & US group \\
\hline & & Raising money to help pay for medical bills & US group \\
\hline & & Raising money to provide patient and caregiver with a dinner date & US group \\
\hline \multirow[t]{7}{*}{ Support from work } & \multirow[t]{3}{*}{ Flexible work hours } & Caregiver is allowed to leave work early & Both groups \\
\hline & & Caregiver can take leave when needed & Both groups \\
\hline & & $\begin{array}{l}\text { Caregiver can cut back on hours, or work more depending on the } \\
\text { situation }\end{array}$ & US group \\
\hline & Flexible work place & Caregiver can work from home & Both groups \\
\hline & Flexible responsibilities & $\begin{array}{l}\text { The duties at work are adjusted because of the mental state of the } \\
\text { caregiver }\end{array}$ & Dutch group \\
\hline & \multirow[t]{2}{*}{ Work-based formal health services } & Counselling service for mental support & Dutch group \\
\hline & & Company doctor & Dutch group \\
\hline
\end{tabular}

have a good informal support system; not knowing where to find local services with expertise in neuro-oncology; the support offered was not a good fit given specific neuro-oncological issues; not qualifying for support when issues were not grave enough; and costs and lack of health insurance coverage. Some caregivers said they had developed their own coping mechanisms such as exercising regularly, thinking positively, counting blessings, and living life 1 day at a time.

\section{Attitudes toward keeping track of care issues over time}

\section{General attitude}

Caregivers generated several possible advantages of keeping track of care issues over time. It could: help them gain insight into their own needs and growing burden, so that they might get help in time; give them a sense of being in control of the situation; help them be more perceptive of changes in the patient's symptoms; and some believed that keeping track of issues could help them process grief. 
Disadvantages were also mentioned. Some caregivers said they do not like keeping track of things per se and many thought that just keeping track would not benefit them. Another disadvantage frequently mentioned by caregivers is that it takes time and requires discipline:

'I don't know if there's a disadvantage, other than the fact that it's time consuming. It takes time to do that and it takes discipline. You have to have discipline to sit down and journal. [...] And this, I look at this company, forget whether it's health related, if we ask people to journal, so they knew what they were spending their time on and what was most important to them during the day, we probably get about 5\% compliance. It's just, that's a hard thing to do on a regular basis.'

Husband of GBM patient who is currently under treatment

Moreover, a few caregivers indicated that it could be difficult to be confronted with their situation frequently. Some caregivers clearly indicated that keeping track of their issues and needs would not be for them. However many were uncertain about their future needs and care issues and emphasized that keeping track of care issues systematically would be most useful around the time when many needs are present. For most, this included the periods immediately post-diagnosis and around disease progression.

\section{Frequency and timing}

Preferences for the frequency of monitoring care issues varied widely, ranging from once a week to once every 18 months. Many indicated that their preferred frequency would depend on the needs they experience and that it would therefore be good to start monitoring shortly after the patient is diagnosed. In terms of timing and setting, most caregivers indicated it would be best for them to complete questions at home, before visiting the hospital. This would allow more time under less stressful circumstances, and lead to more honest answers because of better privacy. Moreover, as not all caregivers were always able to accompany the patient to clinic, this would allow them to keep track of their care issues and needs more frequently. Others however, felt their often long waiting time in the hospital could be put to good use by completing questions then and there.

\section{Format}

Presented with different options to keep track of their issues over time, only a few caregivers preferred a yes/no type checklist as it is easy to complete and unambiguous, while the majority of caregivers believed a multi-item scale would reflect their situation better:

'This is just a concrete yes or no. And it could be like oh I'm having a little bit of a problem with child care or I' $m$ in desperate need of help. And this kind of tells you how big of a problem each individual thing is.' Wife of oligodendroglioma grade II patient with suspected disease progression

A few suggested that a diary form would be best, or alternatively to allow room for free text remarks. Some emphasized that any questionnaire should be as concise as possible to reduce burden. Although caregivers said a paper-based checklist (DT) would be quick and easy to complete, includes relevant topics and would give a good overview of the issues that are present in one glance, they were unsure how they would benefit from completing the checklist:

'Will it provide some way to foresee down times and be able to... You know, help reduce the stress to look for a way to kinda work around it? I don't know, I'm not sure that I could... That it would have like a lot more value than 'hmm that's interesting'. Would it really, would I find a way to make it a less stressful time? You know based on that? I'm not sure it would.'

Husband of patient with medulloblastoma who is receiving treatment

Caregivers indicated that personal feedback from a health care professional would help, but some participants felt this would not be realistically achievable in clinical practice. Although many caregivers mentioned that an eHealth monitoring instrument that comprises a touchscreen system based in the clinic (OQ) would not be ideal as it is not mobile, they did feel completing the questions in this digital manner would be easier and faster than on paper, and better for the environment. Several caregivers said the fully digital option comprising a home-based monitoring instrument (OK) could serve as a guide to supportive care options as the resources it generates are suggested by a trusted source (i.e., the hospital). The opportunity to complete the questions at home and the flexibility in terms of timing were mentioned as important advantages:

'I like it 'cause you could do it at your house, whenever you want to, privacy of your home, be comfortable, and you know. It'd just be you and the computer. I like that.'

Wife of patient with astrocytoma grade II who is under treatment

Negative points raised regarding the fully digital option (OK) were the expected high investment of time, 
an expected need for reminders, and the lack of personal contact.

Table 3 shows caregivers' preferences regarding paperbased and eHealth monitoring systems in general. EHealth monitoring instruments were regarded as having high accuracy and comfortable levels of privacy when completing personal questions. The anonymity that comes with computer use was seen as an advantage especially when experiencing high levels of distress, as it can make expressing their true feelings easier. Important tips for the development of eHealth instruments provided by Dutch caregivers were: to limit the information generated to avoid an information overload, and to automatically save completed questions to reduce frustration if the system crashes (see Table 3). Several caregivers indicated that mobile solutions that allow use on tablets or smartphones would be superior to desktop only instruments. Stand-alone eHealth instruments such as OK were regarded as sufficient when few needs or issues are present, and adding the option to contact a health care professional either digitally, by phone or in person if needed was suggested by several caregivers.

'So I think if you maybe had the answers like the short answers and then give the opportunity to say tell me what you meant by this. And then it will all come out. To give them the opportunity to not be hit by it all of a sudden.'

Wife of oligodendroglioma grade II patient with suspected disease progression

Almost all caregivers felt able to use an eHealth instrument and most, but not all, caregivers opted for either a fully digital instrument or a combination of a digital instrument with personal feedback. A few caregivers, on the other hand, preferred a paper-based checklist with personal feedback instead, and one caregiver suggested to make all options available so that all personal preferences may be reflected.

\section{Conditions for use}

Caregivers made several suggestions for the development of any monitoring instrument, see Table 3. Many said they would not make use of an instrument unless they perceive benefits for themselves; this could be related to the needs present, or to the options for supportive care that are available and affordable. Participants said that any needs assessment should be quick to complete, preferably under $10 \mathrm{~min}$, and be clear and easy to use. Furthermore they indicated that the resources that are referred to, should be tailored specifically to the neuro-oncology situation. Many believed recommendation by a trusted source such as the treatment team would help them to start using a monitoring instrument but it was expected that an incentive (either the presence of supportive care needs, a small monetary reward, or frequent reminders) would be needed to ensure continued use:

'I think some people would say yeah that's a good thing, the doctor's telling me that. There's other people who'll forget about it once they're in the car. There has to be constant reminders. [...] Just an idea, some kind of marketing pushout to people on a regular basis.'

Husband of patient with medulloblastoma who is under treatment

Other tips generated by US caregivers include that any computerized option should be flexible enough to use on different personal devices as public computers were generally not trusted, and should not take up too much of the device's working memory as a slow computer would put them off.

\section{Discussion}

This qualitative study is an extension of our earlier report on PBT caregivers' attitudes and preferences toward monitoring care issues [12]. Although PBT caregivers reported to have utilized a range of different formal and informal support services, those who experienced more care issues indicated to have preferred more support. Appropriate and available supportive care services are, however, difficult for caregivers to find, underlining the important contribution to signposting a monitoring instrument could have. Regarding keeping track of care issues over time, the present study findings from the interviews with family caregivers from the US, support and enrich the results from our previous publication. Similar advantages and disadvantages of keeping track of care issues were mentioned. On the one hand, PBT caregivers feel it can provide more insight into one's own needs and through this avenue, it could help them find their way to supportive care. On the other hand, it takes valuable time and requires discipline, and it can be difficult to face seeing their care issues increase over time. Importantly, PBT caregivers would only find it useful to keep track of their care issues when there are needs present. They commonly felt a short and easy-to-use 'blended care' instrument, that combines digital monitoring with some kind of personal feedback, was most appropriate for them. Furthermore, most caregivers would prefer to complete questions at home, stating that the clinic visit is very stressful and not a good indicator of overall stress. Several useful tips were generated by PBT caregivers that could facilitate instrument development.

The preference for a blended care option corroborated findings from studies among cancer patients in general [21] 
Table 3 Caregivers' preferences for paper-based, digital or blended care monitoring instruments

\begin{tabular}{|c|c|c|c|}
\hline Topics & Key issues & Themes & Participant groups \\
\hline \multirow[t]{17}{*}{ Type of monitoring instrument } & \multicolumn{3}{|l|}{ Preference for... } \\
\hline & \multirow[t]{3}{*}{ Paper-based with personal feedback } & It is quick and easy to complete & Both groups \\
\hline & & $\begin{array}{l}\text { Would provide a quick overview of the } \\
\text { needs present }\end{array}$ & Dutch group \\
\hline & & Not interested in using computers & US group \\
\hline & \multirow[t]{8}{*}{ Fully digital } & $\begin{array}{l}\text { Can be completed at home, which is } \\
\text { comfortable, allows privacy, and time } \\
\text { to focus }\end{array}$ & Both groups \\
\hline & & $\begin{array}{l}\text { Feedback on needs and issues is pro- } \\
\text { vided instantly }\end{array}$ & Both groups \\
\hline & & $\begin{array}{l}\text { Professional advice for referral to sup- } \\
\text { portive care is provided instantly }\end{array}$ & Both groups \\
\hline & & $\begin{array}{l}\text { The data and feedback are trusted to be } \\
\text { accurate }\end{array}$ & Both groups \\
\hline & & It is always accessible, flexible & Both groups \\
\hline & & $\begin{array}{l}\text { Allows you to go back to the advice to } \\
\text { read at leisure }\end{array}$ & Dutch group \\
\hline & & $\begin{array}{l}\text { Requires less action than other meth- } \\
\text { ods-you just complete question- } \\
\text { naires once and it's done }\end{array}$ & US group \\
\hline & & $\begin{array}{l}\text { Saves paper, probably cheaper in long } \\
\text { run }\end{array}$ & US group \\
\hline & \multirow[t]{5}{*}{$\begin{array}{l}\text { Blended care (i.e. digital with personal } \\
\text { contact) }\end{array}$} & $\begin{array}{l}\text { Digital option would suffice when there } \\
\text { is no great need for support; add- } \\
\text { ing personal contact would only be } \\
\text { required when needs arise }\end{array}$ & Both groups \\
\hline & & $\begin{array}{l}\text { Might suffice to just add the option } \\
\text { to actively contact someone if need } \\
\text { arises }\end{array}$ & Both groups \\
\hline & & $\begin{array}{l}\text { Personal contact can help verbalize } \\
\text { issues }\end{array}$ & US group \\
\hline & & $\begin{array}{l}\text { Personal contact provides extra sup- } \\
\text { port-talking can help to relieve } \\
\text { stress }\end{array}$ & US group \\
\hline & & It is more interactive & Both groups \\
\hline \multirow[t]{8}{*}{ Reasons for (non)use of any instrument } & \multirow[t]{2}{*}{ Perceived as beneficial } & $\begin{array}{l}\text { Most likely to use if there are needs } \\
\text { present }\end{array}$ & Both groups \\
\hline & & $\begin{array}{l}\text { Would only continue to use if there is } \\
\text { perceived benefit }\end{array}$ & Both groups \\
\hline & Clarity & Should be clear and easy-to-use & Both groups \\
\hline & \multirow[t]{2}{*}{ Investment of time } & $\begin{array}{l}\text { The likeliness to use an instrument is } \\
\text { dependent on the time it takes }\end{array}$ & Both groups \\
\hline & & $\begin{array}{l}\text { Should not take more than } 10 \mathrm{~min} \text { to } \\
\text { complete questions }\end{array}$ & US group \\
\hline & \multirow[t]{3}{*}{ Available resources } & $\begin{array}{l}\text { Would only be useful if supportive care } \\
\text { is actually available to them }\end{array}$ & Both groups \\
\hline & & $\begin{array}{l}\text { Resources should fit neuro-oncology } \\
\text { specific needs }\end{array}$ & Both groups \\
\hline & & $\begin{array}{l}\text { Guide toward good information, good } \\
\text { supportive care options, and good } \\
\text { stress-relieve techniques }\end{array}$ & US group \\
\hline
\end{tabular}


Table 3 (continued)

\begin{tabular}{|c|c|c|c|}
\hline Topics & Key issues & Themes & Participant groups \\
\hline & Recommendation by treatment team & $\begin{array}{l}\text { Would not seek out a monitoring instru- } \\
\text { ment without recommendation by } \\
\text { treatment team }\end{array}$ & Both groups \\
\hline & & $\begin{array}{l}\text { Would help recognize that it is not just } \\
\text { another internet resource }\end{array}$ & Dutch group \\
\hline & & $\begin{array}{l}\text { Recommendation would help as incen- } \\
\text { tive to start only }\end{array}$ & Both groups \\
\hline & Continued use & $\begin{array}{l}\text { An extra incentive is necessary for peo- } \\
\text { ple to continue to keep track: money } \\
\text { or a personal coach }\end{array}$ & US group \\
\hline & & $\begin{array}{l}\text { Reminders are needed to continue use, } \\
\text { especially when things are going well }\end{array}$ & Both groups \\
\hline & & $\begin{array}{l}\text { A personal (face-to-face) reminder for } \\
\text { use can help }\end{array}$ & US group \\
\hline \multirow[t]{19}{*}{$\begin{array}{l}\text { Reasons for (non) use of digital instru- } \\
\text { ments }\end{array}$} & Anonymity and privacy & $\begin{array}{l}\text { Easier to be honest about your feelings } \\
\text { when you feel like you're by yourself }\end{array}$ & Both groups \\
\hline & & $\begin{array}{l}\text { Easier to answer questions presented } \\
\text { digitally when stressed }\end{array}$ & US group \\
\hline & & $\begin{array}{l}\text { Privacy is a prerequisite; can be difficult } \\
\text { to answer questions when patient is } \\
\text { looking over your shoulder }\end{array}$ & Dutch group \\
\hline & Accuracy of digital data & $\begin{array}{l}\text { Data is accurate and easy to quantify for } \\
\text { clinicians/researchers }\end{array}$ & US group \\
\hline & Limit information & $\begin{array}{l}\text { Presenting one question at a time can } \\
\text { help to retain focus }\end{array}$ & Dutch group \\
\hline & & $\begin{array}{l}\text { Be wary of information overload on } \\
\text { computer systems }\end{array}$ & Dutch group \\
\hline & Automatic saving & $\begin{array}{l}\text { Can help to save answers frequently and } \\
\text { automatically }\end{array}$ & Dutch group \\
\hline & Computer and internet access & $\begin{array}{l}\text { Would not use the instrument on a } \\
\text { public computer }\end{array}$ & US group \\
\hline & & Slow computer would discourage use & US group \\
\hline & Mobile solutions & $\begin{array}{l}\text { Mobile solutions might be better than } \\
\text { PC-based ones }\end{array}$ & Both groups \\
\hline & & Apps are always available & US group \\
\hline & Computer literacy & Good to very good computer literacy & Both groups \\
\hline & & $\begin{array}{l}\text { Can use the computer for 'simple } \\
\text { things', including google, home shop- } \\
\text { ping and banking }\end{array}$ & Both groups \\
\hline & & $\begin{array}{l}\text { Can use the computer but is not very } \\
\text { used to it }\end{array}$ & US group \\
\hline & & Not one to sit in front of a computer & Both groups \\
\hline & & $\begin{array}{l}\text { Not computer literate, can't even turn } \\
\text { it on }\end{array}$ & US group \\
\hline & & $\begin{array}{l}\text { Most elderly people can also use the } \\
\text { computer nowadays }\end{array}$ & US group \\
\hline & & Can be difficult for older people & US group \\
\hline & Alternatives & $\begin{array}{l}\text { For those not computer literate, a paper } \\
\text { questionnaire might be used as an } \\
\text { alternative }\end{array}$ & US group \\
\hline
\end{tabular}


and neuro-oncology patient/caregiver populations in particular [22]. Cancer survivors $(N=212)$ viewed eHealth applications favorably, with $48 \%$ indicating that they would find the option to self-monitor their side-effects and symptoms attractive [21]. A feasibility study testing a Danish brain tumor website among brain tumor patients and caregivers furthermore showed that especially caregivers appreciated the option to contact health care professionals online [22]. However, our report focuses on caregivers' preferences for keeping track of their own care issues over time - which is, indeed, a relatively unexplored area [23, 24].

Our findings confirm that the preferences and attitudes of family caregivers in neuro-oncology are likely not heavily influenced by country- or culture-specific differences. Of course, differences in the experiences and satisfaction with supportive care have been observed between US and Dutch PBT caregivers. Any caregiver monitoring instrument could take these differences into account to ensure advice on supportive care services meets the needs. Religion and religious communities as well as practical and financial aid from community members played a larger role in the support system of US caregivers, whereas Dutch caregivers more frequently reported having received formal support from the work place, legal advice, and transportation services. This reflects differences between the two countries in terms of health care organization [25-27] and (religious) culture [28, 29]. However, the experienced care issues and unmet supportive care needs expressed were similar. Therefore, the development of any instrument has the potential to benefit a large group of family caregivers. It would however, remain interesting to interview family caregivers from less developed countries or from non-Western cultures as well, to see if results hold.

A limitation of this study is that not all interviews were performed by the same person, which could potentially have influenced study outcomes. Rather, a research psychologist involved in both research groups (FB) completed (or in one case, supervised) the US interviews whereas the Dutch interviews were conducted by a trained and registered clinical psychologist (KH). To minimize the possible influence of this change in interviewers, the semi-structured interview schedule was translated directly from Dutch to English and the same examples of monitoring instruments were used. For practical reasons we chose to only recruit participants who could either be interviewed in the clinic or lived within a $3 \mathrm{~h}$ drive from Pittsburgh. This radius was also chosen because it includes both urban and rural areas and has resulted in a good and varied representation of participants in our previous studies. The US and Dutch samples showed some differences in composition, with the US group including a greater variety of tumor types and the
Dutch sample including fewer male caregivers. Combined, these groups are a good representation of the population, however the differences between the groups might have contributed to some of the differences described in this report. Where our previous report focused on participants' thoughts on specific existing monitoring instruments, here, we used the same instruments as examples but aimed to provide broader recommendations for future instrument development. This is necessary as at present, there is no (neuro-oncology) caregiver-specific monitoring instrument available in clinical practice. Although several interventions have been performed to try and provide support for family caregivers in neuro-oncology, some utilizing eHealth or telehealth solutions [30], none so far include the option to keep track of care issues over time to only provide supportive care as needed. Caregivers indicated they would only want to use a monitoring instrument when they experience care issues. Predicting when issues will arise is, however, very difficult for both health care professionals and caregivers, so any monitoring instrument would have to be as short as possible to minimize the added burden and improve compliance. The suggestions and ideas generated by caregivers can serve as pointers in adapting and optimizing any evidence-based instrument for use by family caregivers in neuro-oncology.

To summarize, we found that two separate samples of family caregivers in neuro-oncology prefer a blended care option that combines digital monitoring with personal contact. Adapting and implementing a blended care instrument to keep track of issues and supportive care needs over time has the potential to benefit large groups of family caregivers in neuro-oncology.

Acknowledgements This study was supported by a Niels Stensen Fellowship and a Yorkshire Cancer Research University Academic Fellowship. We would like to thank the patients and family caregivers who participated in this study, as well as the clinicians and nurse practitioners for helping us with recruitment. Furthermore, many thanks to Julianne Kingsley for her help in data transcription and to Natalia Sak-Dankosky for her efforts in recruitment. Finally, many thanks to Wilmy Cleijne for helping with data analysis of the Dutch caregiver interviews. We wish you a pleasant retirement!

Open Access This article is distributed under the terms of the Creative Commons Attribution 4.0 International License (http:// creativecommons.org/licenses/by/4.0/), which permits unrestricted use, distribution, and reproduction in any medium, provided you give appropriate credit to the original author(s) and the source, provide a link to the Creative Commons license, and indicate if changes were made.

\section{References}

1. DeAngelis LM (2001) Brain tumors. N Engl J Med 344(2):114-123 
2. Rooney AG, Netten A, McNamara S et al (2014) Assessment of a brain-tumour-specific Patient Concerns Inventory in the neurooncology clinic. Support Care Cancer 22(4):1059-1069

3. Mukand JA, Blackinton DD, Crincoli MG et al (2001) Incidence of neurologic deficits and rehabilitation of patients with brain tumors. Am J Phys Med Rehabil 80(5):346

4. Taphoorn MJB, Klein M (2004) Cognitive deficits in adult patients with brain tumors. Lancet Neurol 3(3):159-168

5. Boele FW, Klein M, Reijneveld JC et al (2014) Symptom management and quality of life in glioma patients. CNS Oncol 3(1):37-47

6. Sterckx W, Coolbrandt A, Dierckx de Casterle B et al (2013) The impact of a high-grade glioma on everyday life: a systematic review from the patients and caregivers perspective. Eur J Oncol Nurs 17(1):107-117

7. Choi CW, Stone RA, Kim KH et al (2012) Group-based trajectory modeling of caregiver psychological distress over time. Ann Behav Med 44(1):73-84

8. Sherwood PR, Given BA, Donovan H et al (2008) Guiding research in family care: a new approach to oncology caregiving. Psycho-Oncology 17(10):986-996

9. Mitchell AJ (2013) Screening for cancer-related distress: when is implementation successful and when is it unsuccessful? Acta Oncol 52(2):216-224

10. van Gemert-Pijnen JE, Nijland N, van Limburg M et al (2011) A holistic framework to improve the uptake and impact of eHealth technologies. J Med Internet Res 13(4):e111

11. Nijland N, van Gemert-Pijnen JE, Kelders SM et al (2011) Factors influencing the use of a Web-based application for supporting the self-care of patients with type 2 diabetes: a longitudinal study. J Med Internet Res 13(3):e71

12. Boele F, van Uden-Kraan C, Hilverda K et al (2016) Attitudes and preferences towards monitoring supportive care needs in glioma patients and their informal caregivers. Support Care Cancer 24(7):3011-3022

13. Boele FW, Klein M, Armstrong T et al (2014) Needs of informal caregivers of primary malignant brain tumor patients in the United States and the Netherlands: an international comparison. Neuro-Oncology 16(Suppl 2):ii20-ii

14. Francis J, Johnston M, Robertson C et al (2015) What is an adequate sample size? Operationalising data saturation for theorybased interview studies. Psychol Health 25(10):1229-1245

15. Zwahlen D, Hagenbuch N, Carley MI et al (2008) Screening cancer patients' families with the distress thermometer (DT): a validation study. Psycho-Oncology 17(10):959-966
16. Cnossen IC, de Bree R, Rinkel RN et al (2012) Computerized monitoring of patient-reported speech and swallowing problems in head and neck cancer patients in clinical practice. Support Care Cancer 20(11):2925-2931

17. Duman-Lubberding S, van Uden-Kraan C, Peek N et al (2015) An eHealth application in head and neck cancer survivorship care: health care professionals' perspectives. J Med Internet Res 17(10): 235

18. Braun V, Clarke V (2006) Using thematic analysis in psychology. Qual Res Psychol 3(2):77-101

19. Joffe H, Yardley L (2004) Content and thematic analysis. In: Marks D, Yardley L (eds) Research methods for clinical and health psychology. SAGE Publications, p 56

20. Tong A, Sainsbury P, Craig J (2007) Consolidated criteria for reporting qualitative research (COREQ): a 32-item checklist for interviews and focus groups. Int $\mathrm{J}$ Qual Health Care 19(6):349-357

21. Jansen F, van Uden-Kraan C, van Zwieten V et al (2015) Cancer survivors' perceived need for supportive care and their attitude towards self-management and eHealth. Support Care Cancer 23(6):1679-1688

22. Piil K, Jakobsen J, Juhler M, Jarden M (2015) The feasibility of a brain tumour website. Eur J Oncol Nurs 19(6):686-693

23. Maqbool T, Agarwal A, Sium A et al (2016) Informational and supportive care needs of brain metastases patients and caregivers: a systematic review. J Cancer Educ. doi:10.1007/ s13187-016-1030-5

24. Slev V, Mistiaen P, Pasman H et al (2016) Effects of eHealth for patients and informal caregivers confronted with cancer: a metareview. Int J Med Informatics 87:54-67

25. van Weel C, Schers H, Timmermans A (2012) Health care in the Netherlands. J Am Board Fam Med 25(Suppl 1):S12-S17

26. Schoen C, Osborn R, Doty M et al (2007) Toward higher-performance health systems: adults' health care experiences in seven countries, 2007. Health Aff 26(6):w717-w734

27. Obama B (2016) United States health care reform: progress to date and next steps. JAMA 316(5):525-532

28. Berghuijs J, Pieper J, Bakker C (2013) Being 'spiritual' and being 'religious' in Europe: diverging life orientations. J Contemp Relig 28(1):15-32

29. Ellison CG, McFarland MJ (2013) The social context of religion and spirituality in the United States. APA Handb Psychol Relig Spiritual 1:21-50

30. Boele F, Grant R, Sherwood P (2016) Challenges and support for family caregivers of glioma patients. Br J Neurosci Nurs 13:8-16 